\title{
Changes in Retinal Perfusion in Leber's Hereditary Optic Neuropathy: An Optical Coherence Tomography-Angiography Study
}

\author{
Jian Yu ${ }^{a, b, c}$ Huan Xu ${ }^{a, b, c}$ Yongheng Huang ${ }^{a, b, c, d}$ Ruiping Gu ${ }^{a, b, c}$ \\ Yuan Zong a,b,c Haohao Zhu ${ }^{e}$ Min Wang ${ }^{a, b, c}$
}

aEye Institute and Department of Ophthalmology, Eye \& ENT Hospital, Fudan University, Shanghai, China;

${ }^{b}$ Key Laboratory of Myopia of State Health Ministry, and Shanghai Key Laboratory of Visual Impairment and Restoration, Shanghai, China; ${ }^{C} \mathrm{NHC}$ Key Laboratory of Myopia (Fudan University), Key Laboratory of Myopia, Chinese Academy of Medical Sciences, Shanghai, China; ${ }^{d}$ Department of Ophthalmology, Kiang Wu Hospital, Macau Special Administration Region, Macau, China; 'Department of Ophthalmology, People's Hospital of Shanghai No. 5, Shanghai, China

\section{Keywords \\ Leber's hereditary optic neuropathy · Optical coherence tomography-angiography · Radial peripapillary capillary · Parafoveal superficial capillary plexus · Vessel density}

\begin{abstract}
Introduction: We aimed to study the change in the retinal perfusion in Leber's hereditary optic neuropathy (LHON). Methods: Fourteen patients (28 eyes) diagnosed with LHON and 14 healthy controls ( 28 eyes) were enrolled. The retinal vessel densities in the parafoveal and peripapillary areas were measured with optical coherence tomography-angiography. Results: In the subacute LHON patients, the parafoveal superficial capillary plexus (SCP) and inner retinal thickness (IRT) were significantly reduced in all sectors compared with the controls (all $p<0.05$ ), and the radial peripapillary capillary (RPC) network was significantly reduced in the temporal and inferior temporal sectors compared with the controls $(p<0.05)$. In the chronic LHON patients, the SCP and IRT were significantly lower in all sectors than in the controls (all $p<0.05$ ), the RPC vessel density and thickness were significantly lower in all sectors than in the controls and lower in the temporal, superior temporal, inferior temporal, and
\end{abstract}

karger@karger.com www.karger.com/ore

Karger"
(C) 2021 The Author(s)

Published by S. Karger AG, Basel

This is an Open Access article licensed under the Creative Commons Attribution-NonCommercial-4.0 International License (CC BY-NC) (http://www.karger.com/Services/OpenAccessLicense), applicable to the online version of the article only. Usage and distribution for commercial purposes requires written permission. nasal sectors than in the subacute-stage patients (all $p<$ 0.05). Conclusion: The retinal structure and the perfusion of the macular and peripapillary areas are reduced in subacute LHON, and the retinal structure and the perfusion of the peripapillary area are further reduced in chronic LHON.

(C) 2021 The Author(s).

Published by S. Karger AG, Basel

\section{Introduction}

Leber's hereditary optic neuropathy (LHON) is a mitochondrial disease caused by point mutations in the maternally inherited mitochondrial DNA (mtDNA) that affect complex I of the oxidative phosphorylation chain [1-3]. It was first described in 1871 by Leber [4]. The 3 most frequent pathogenic mutations in LHON are at nucleotidepositions11778/ND4,3460/ND1, and14484/ND6. LHON typically occurs in late childhood and early adulthood, with little or no propensity for recovery, and is remarkable in its tissue selectivity insofar as this disorder is

Jian Yu and Huan Xu contributed equally as first authors. Wenghang Wong is the spelling of Yongheng Huang used in the Macau Special Administration Region, People's Republic of China. 

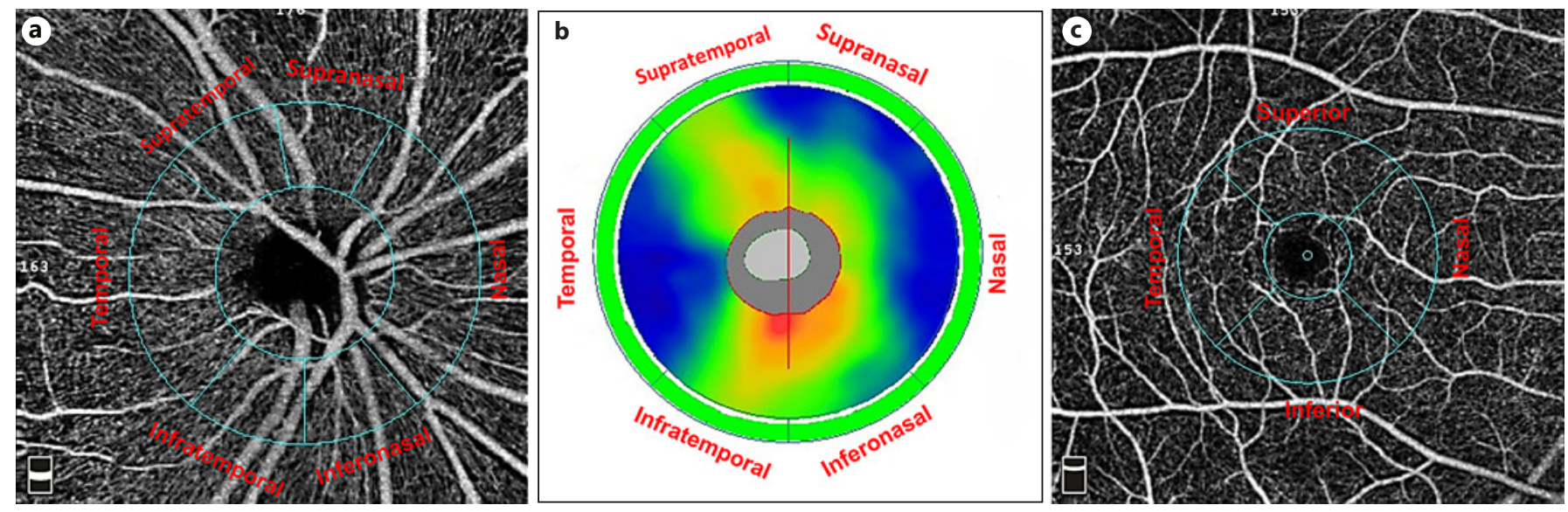

Fig. 1. Segmentation of the RPC, pRNFL, parafoveal SCP, and IRT. RPC (a); pRNFL (b); Parafoveal SCP and IRT (c). SCP, superficial capillary plexus; IRT, inner retinal thickness; pRNFL, peripapillary retinal nerve fiber layer; RPC, radial peripapillary capillary.

mainly limited to the retinal ganglion cells (RGCs) and their axons in the optic nerve $[1-3,5]$. Using optical coherence tomography (OCT), several studies have shown that the thinning of the macular ganglion cell-inner plexiform layer (GCIPL) and the peripapillary retinal nerve fiber layer ( $\mathrm{pRNFL}$ ) is detectable after symptom presentation $[6,7]$. Morphological changes in the retinal vessels that supply the RGCs and their axons have also been detected in patients with LHON, such as circumpapillary telangiectatic microangiopathy and small vessel tortuosity [8-10]. However, the role of the quantitative microvascular supply in the pathogenesis of LHON is poorly understood. Recent developments in optical coherence tomography-angiography (OCTA) allow reproducible quantitative measurements of the retinal vascular vessel density to be made in different regions [11-13]. In this study, we measured the changes in retinal perfusion in patients with LHON and evaluated the association between retinal perfusion and RGC damage in LHON patients.

\section{Methods}

\section{Participants}

In this cross-sectional observational study, patients with a clinically and molecularly confirmed diagnosis of LHON at the Eye \& ENT Hospital of Fudan University, Shanghai, China, between May 2015 and September 2016 were enrolled. The study was approved by the Ethics Committee of the Eye and ENT Hospital of Fudan University and performed in accordance with the Declaration of Helsinki and its amendments. Signed informed consent was obtained from all the participants. All subjects underwent compre- hensive ophthalmic examinations, which included best-corrected visual acuity (BCVA), examination with slit-lamp biomicroscopy, refraction measurements with an autorefractor, the calculation of the spherical equivalence based on the spherical diopter plus onehalf of the cylindrical dioptric power, a dilated fundus examination, and the measurement of intraocular pressure with a noncontact tonometer. The central visual field was assessed with a Humphrey field analyzer (750, Carl Zeiss Meditec, Jena, Germany) with a Swedish interactive thresholding algorithm standard 30-2 test program (Carl Zeiss Meditec), and the mean deviation (MD) was determined.

To be included, patients were required to have experienced visual loss at least 3 months before enrollment with no obvious optic disc edema, as determined by a neuro-ophthalmologist (MW). The exclusion criteria were the presence of any retinal pathology and/or optic nerve disease other than LHON, the presence of diabetes mellitus, hypertension, migraine, or other systemic disease. The patients were divided into 2 groups based on their symptom duration (1) patients in the late subacute stage of LHON, with symptom presentation in the previous $4-6$ months (late subacute LHON); or (2) patients in the chronic stage of the disease, with symptom presentation $>12$ months previously (chronic LHON). Fifteen sex- and age-matched healthy volunteers were recruited as the control group.

\section{OCTA Acquisition and Processing}

Images of both eyes were acquired on the same visit. The OCTA scans were made with a spectral domain system (RTVueXR Avanti, Optovue, Fremont, CA, USA). The details of the OCTA system have been described in a previous study $[14,15]$. The vessel densities were calculated as the percentage areas occupied by large vessels and microvessels [16] and were automatically determined with the system software. The quality of the OCTA images was evaluated by 2 independent ophthalmologists blinded to the participants' diagnostic categories and groups. Poor-quality images with a signal strength index of $<40$ were rejected. 
Table 1. Clinical data and OCT parameters of LHON patients and controls at different stages

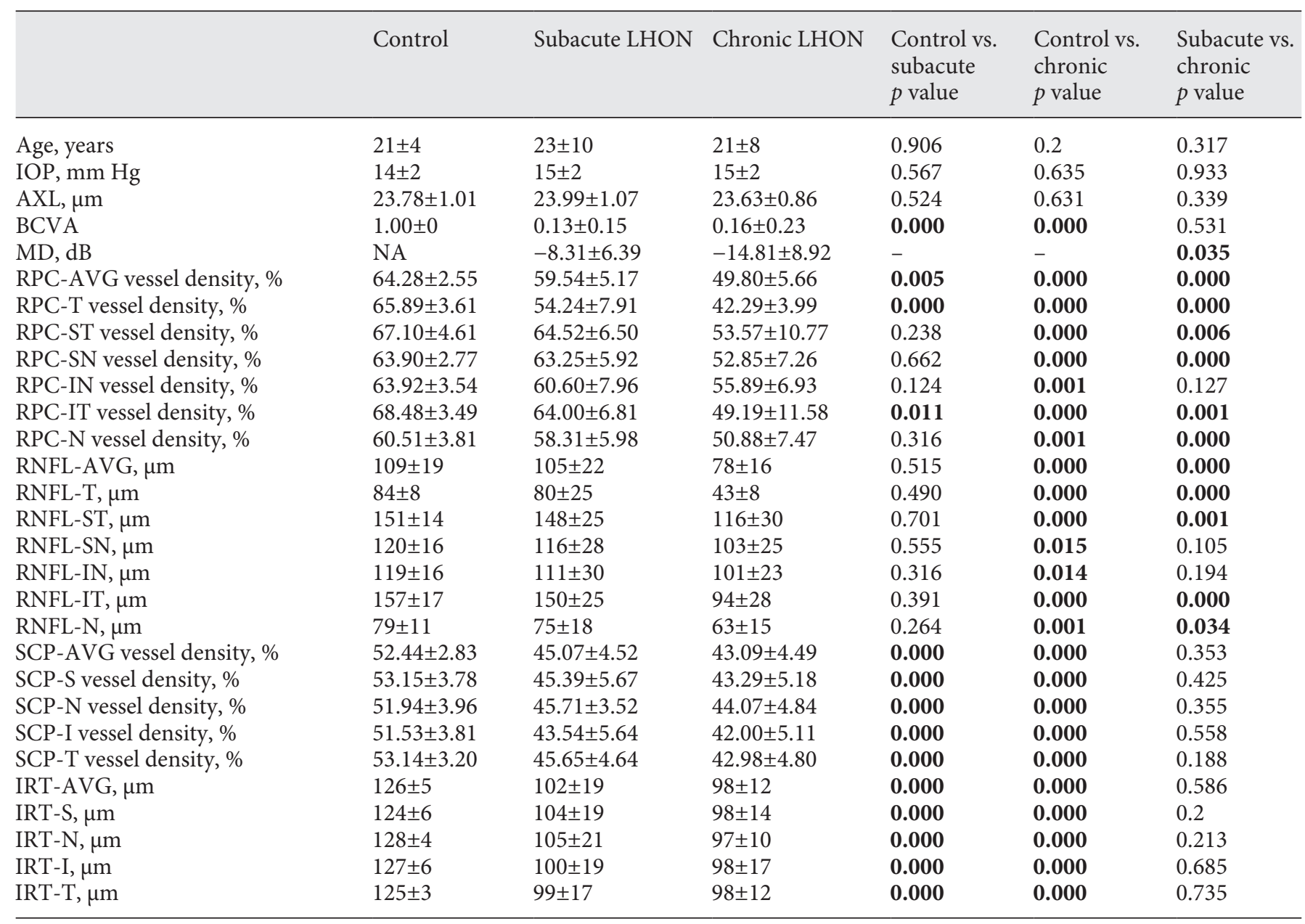

LHON, Leber's hereditary optic neuropathy; BCVA, best-corrected visual acuity; MD, mean deviation; dB, decibel; RPC, radial peripapillary capillary; SCP, superficial capillary plexus; RNFL, retinal nerve fiber layer; AVG, average; T, temporal; S, superior; I: inferior; N, nasal; AXL, axial length; IOP, intraocular pressure; IRT, inner retinal thickness; OCT, optical coherence tomography.

The radial peripapillary capillary (RPC) network was visualized on the scans within a $0.75-\mathrm{mm}$-wide elliptical annular region extending from the optic disc boundary and was divided into 6 sectors (Fig. 1a) based on the Garway-Heath map [17]. The average and sectorial RPC vessel densities within the internal limiting membrane and the nerve fiber layer were automatically analyzed with the software. The thickness of the RNFL was measured within a circular region with a diameter of $3.45 \mathrm{~mm}$, centered on the optic disc, and the average and sectorial RPC vessel densities were determined (Fig. 1b).

The parafoveal area was defined as an annulus with an outer diameter of $3 \mathrm{~mm}$ and an inner diameter of $1 \mathrm{~mm}$, centered on the fovea and divided into 4 sectors (Fig. 1c). The average and sectorial parafoveal superficial capillary plexus (SCP), extending from $3 \mu \mathrm{m}$ below the internal limiting membrane to the outer boundary of the inner plexiform layer, were analyzed. The average and sectorial parafoveal inner retinal thicknesses (IRTs) were measured from the internal limiting membrane to the outer boundary of the inner plexiform layer using the retinal map protocol.

\section{Statistical Analyses}

All statistical analyses were performed with IBM SPSS V.20.0 (SPSS, Inc., Chicago, IL, USA). The demographic and ophthalmic characteristics of the study groups were compared with the Kruskal-Wallis test or Fisher's exact test. The ophthalmological and OCT parameters were compared among groups with generalized estimating equations (GEEs) to adjust for intrasubject inter-eye differences. The GEE results are reported as regression coefficients $(\beta)$ with standard errors. The correlations between the OCTA parameters and the clinical parameters were determined with the GEE models. Statistical significance was set at $p<0.05$. 


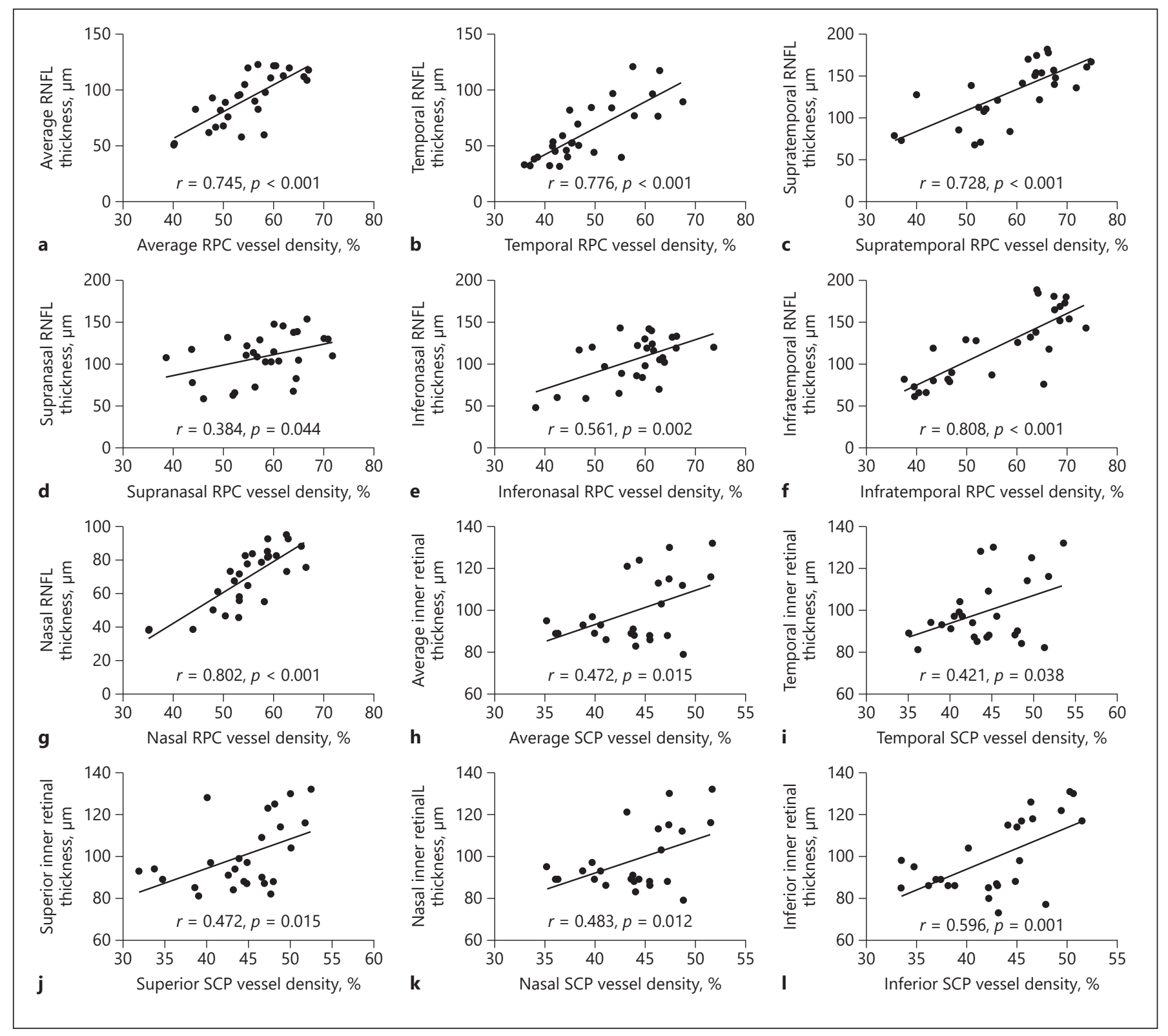

Fig. 2. Correlation between retinal thickness and vessel density in the corresponding areas. Average retinal nerve fiber layer thickness and RPC vessel density (a); temporal retinal nerve fiber layer thickness and RPC vessel density (b); supratemporal retinal nerve fiber layer thickness and RPC vessel density (c); supranasal retinal nerve fiber layer thickness and RPC vessel density (d); inferonasal retinal nerve fiber layer thickness and RPC vessel density (e); inferotem- poral retinal nerve fiber layer thickness and RPC vessel density (f); nasal retinal nerve fiber layer thickness and RPC vessel density $(\mathbf{g})$; average IRT and SCP vessel density (h); temporal IRT and SCP vessel density (i); superior IRT and SCP vessel density (j); nasal IRT and SCP vessel density (k); inferior nasal IRT and SCP vessel density (I). RPC, radial peripapillary capillary; SCP, superficial capillary plexus; RNFL, retinal nerve fiber layer; IRT, inner retinal thickness.

\section{Results}

Fourteen LHON patients were enrolled in the study: 11 patients carried the mtDNA mutation $\mathrm{m} .11778 \mathrm{G}>$ $\mathrm{A} / M T-N D 4,2$ patients carried the m.14484T $>\mathrm{C} / M T$ -

ND6 mutation, and 1 patient carried the m.3460G > A/MT-ND1 mutation. The average duration of symptomatic disease was $18 \pm 30$ months (range 3-120 months) in the LHON patients. Seven LHON patients (14 eyes) were classified in the subacute phase and 7 (14 eyes) in the 
chronic phase. Two OCTA images of the parafoveal area from subacute LHON patients were excluded from the statistical analysis because their quality was poor due to motion artifacts.

The mean age, intraocular pressure, and axial length did not differ significantly among the 3 groups (all $p>$ 0.05 ; Table 1). The BCVAs of the subacute and chronic LHON eyes were lower than those of the control eyes (all $p<0.05$; Table 1). There was no difference in BCVA between the subacute and chronic LHON eyes, but MD was lower in the patients with chronic LHON than in those with subacute LHON. In the subacute LHON eyes, the parafoveal SCP vessel density and IRT were significantly reduced in all sectors compared with those of the control eyes (all $p<0.05$; Table 1 ). The RPC vessel density was significantly lower in the temporal and inferior temporal sectors than in the control eyes $(p<0.05$; Table 1$)$, but the pRNFL thickness did not differ significantly from that in the control eyes in any sector (all $p>0.05$; Table 1 ). In the patients with chronic LHON, the parafoveal SCP vessel density and IRT were significantly lower in all sectors than in the control eyes (all $p<0.05$; Table 1), but did not differ significantly from those of the patients with subacute-stage LHON (all $p>0.05$; Table 1 ). In the eyes with chronic LHON, the RPC vessel density and pRNFL thickness were significantly lower in all sectors than in the control eyes, and were significantly lower in the temporal, superior temporal, inferior temporal, and nasal sectors than in the subacute-stage eyes (all $p<0.05$; Table 1).

The parafoveal vessel density correlated significantly with the IRT in the corresponding areas in all sectors (all $p<0.05$; Fig. 2), and the RPC vessel density correlated significantly with the RNFL thickness in all corresponding areas in all sectors (all $p<0.05$; Fig. 2 ). The BCVA was significantly positively correlated with the parafoveal SCP vessel density $(p<0.05)$ but not with the IRT $(p>$ 0.05 ), and the MD was significantly positively correlated with the RPC vessel density and pRNFL thickness (all $p<$ 0.05 ) in LHON patients.

\section{Discussion}

In this study, we have demonstrated the changes that occur in the structure and perfusion in different retinal regions of LHON eyes during different stages of the disease. The parafoveal SCP and IRT were markedly reduced in all sectors in both the subacute and chronic stages of LHON. However, in the peripapillary area, only the temporal and inferotemporal RPC vessel densities were clear-

Changes in Retinal Perfusion in Leber's

Hereditary Optic Neuropathy ly reduced in subacute LHON, whereas the RPC vessel density and RNFL thickness were significantly reduced in all sectors in the chronic stage. SCP and RPC vessel density also correlated significantly with retinal thickness in the corresponding areas in all sectors (all $p<0.05)$ in the LHON patients.

LHON is characterized by mitochondrial dysfunction, which initially and mainly affects RGCs and their axons $[1,3]$. It has been suggested that RGC damage implies a reduction in the metabolic rate of the retina, accompanied by a corresponding reduction in retinal flow or attempted vascular compensation. Therefore, ophthalmologists have studied the changes in the retinal vessels of LHON patients with a variety of methods, including fundus photography and fundus fluorescein angiography $[9$, 18]. Although these methods provide important clinical information, their use in the clinical setting is limited by their invasiveness and low resolution. For a long time, ophthalmologists only observed the morphological changes in the retinal capillaries of patients with LHON, such as the increase in the tortuosity and size of the capillaries [8-10]. However, OCTA is an effective, noninvasive tool for monitoring blood flow in small retinal vessels, and has been used to detect microvascular changes in other optic neuropathies $[14,19,20]$. Nonetheless, given the rarity of LHON, only a few reports have described the OCTA features of this disease. Only 3 studies and a few case reports have quantitatively evaluated the peripapillary or parafoveal microvascular changes in LHON [21-24]. Kousal et al. [23] and Balducci et al. [21] found that the RPC vessel density in the peripapillary area is reduced in the chronic stage of LHON [21, 23], and Borrelli et al. [22] showed that the parafoveal vessel density in the macular area was lower in 29 eyes of LHON patients than in the controls. In the present study, we found that the RGCs (IRT and RNFL) and the retinal vessels supplying the RGCs (SCP and RPC vessel density) were both reduced in patients with chronic-stage LHON compared with those in the controls. The reduction in retinal vessels seems to accompany the change in the retinal structure, exemplified by the strong correlation between the RPC vessel density and RNFL thickness and the strong correlation between SCP and the IRT. Our results strongly suggest a metabolic relationship between RGCs and the vascular supply, although another mechanism may also account for the reduced vessel density in LHON eyes. Histopathological studies have demonstrated retinal vascular abnormalities in LHON patients, and the postmortem analysis of human capillaries in the optic nerve head revealed mitochondrial proliferation affecting both the 
endothelial and the smooth muscle components of the blood vessel walls in LHON patients [25].

Investigating the changes in the retinal structure and vessels in different stages of LHON can help us to understand the mechanism of the diseases and to determine the therapeutic time window. However, previous studies mainly focused on structural changes [26]. And studies of the vascular changes that occur in the different stages of LHON are limited. A case report by Matsuzaki et al. [24] presented the longitudinal changes in the RPC vessel density and peripapillary RNFL in a patient who presented with acute- and presymptomaticstage LHON in the left and right eyes, respectively. They found that once the temporal RNFL started to decrease in thickness, the defect in the temporal RPC vessel density gradually increased. In the present study, we found that in the subacute stage of LHON eyes, the RPC vessel density was lower in the temporal sector than in the control eyes. However, the RNFL thickness did not differ in the subacute stage. The RNFL thickness, and RPC vessel density decreased further as the disease progressed to the chronic stage of LHON, which is consistent with Balducci et al. [21] The RNFL thickness and RPC vessel density decreased with the MD as the disease progressed, which confirmed our finding that the MD was correlated significantly with the RPC vessel density and pRNFL thickness in LHON patients. Previously, Li et al. [27] reported that visual stimulation increases neural activity and cerebral blood flow under normal physiological conditions, suggesting that perfusion of the central nervous system may be sensitive to changes in neural activity, and Pan et al. [28] found that the papillomacular bundle, which contains the smallest caliber RGC fibers, is most vulnerable to mitochondrial dysfunction. These results may partly explain our findings that the RPC vessel density decreased before the loss of fibers and that vascular attenuation preferentially involved the temporal region of the peripapillary area in subacute LHON. Borrelli et al. [22] showed that the parafoveal vessel density was reduced in the chronic stage, and we found that the parafoveal vessel density and the IRT were reduced in the subacute stage, although no further significant reduction in the vessel density or the IRT was detected thereafter. No other study has investigated the changes in the macular vasculature during the subacute stage, but a longitudinal follow-up study of the GCIPL changes in LHON may support our results. This study found that early GCIPL damage begins about 6 weeks before the onset of visual loss in patients with first eye involvement. No further significant reduction in the thickness of the GCIPL was detected 6 months after visual loss [6].

Two important conclusions can be drawn from our study. First, the subacute (6-month) stage may represent the end of the loss of the IRT and parafoveal vessel density, which suggests that the previous 6 months (from disease onset) is the therapeutic window for use in future clinical trials. Second, the changes in the retinal vessels and the structure of the macula occur before those in the peripapillary area, so monitoring the changes in the structure and vessels may allow us to follow the progress of the disease. Guy et al. [29] demonstrated that visual acuity, the visual field, and the thickness of the RNFL remained stable and within the normal ranges, whereas the mean pattern electroretinography amplitudes of the carriers decreased progressively by $40 \%$ between baseline and 36 months [29], suggesting subclinical RGC dysfunction in asymptomatic LHON subjects. This RGC dysfunction would reduce the metabolic demand, which is expected to reduce the retinal perfusion via autoregulatory mechanisms. Therefore, we propose that the perfusion of the macula is reduced in asymptomatic LHON subjects, which must be tested in a future study of asymptomatic LHON patients.

In addition to retinal structural indices, retinal blood flow has also been recognized as a potentially valuable biomarker for neurodegenerative optic neuropathies [21, 30-32]. OCTA has been a transformative tool for mapping vascular perfusion at the structural and qualitative functional levels and it has been used to detect microvasculature change in many neurodegenerative optic neuropathies. Superficial vessels may be more impaired in primary open-angle glaucoma, whereas the deeper layers of the retina are more affected in Alzheimer's disease [30]. Kwapong et al. [31] reported reduced microvascular density in most areas of the whole retina. In early primary open-angle glaucoma, significant microvascular damage was present in both the macular and peripapillary areas [32]. In our study, we found that the parafoveal SCP and IRT were markedly reduced in all sectors, but only the temporal and inferotemporal RPC vessel densities were clearly reduced in subacute LHON. However, we also need to recognize that the OCTA findings in retinal vasculature have a significant amount of overlap within neurodegenerative disorders, and thus it is challenging to describe the contributions of each pathological entity accurately. Consequently, larger prospective longitudinal studies are required.

Our study had several limitations. First, our sample size was relatively small, which was expected and un- 
avoidable given the rarity of LHON. Therefore, independent validation of our results with other cohorts is particularly important. Second, the cross-sectional nature of the study entailed limitations, and a longitudinal study might document the dynamic changes over time, thus confirming the hypothesized scenario. Another limitation was related to the genetic heterogeneity of the patients studied. No meaningful stratification by mutation was performed because the $11778 \mathrm{G}>\mathrm{A} / \mathrm{MT}$-ND4 mutation was overrepresented.

Overall, our study shows that the retinal structure and the perfusion of the macular area is reduced prior to that of the peripapillary area, but did not decrease further after the subacute stage. Measurements of the changes in the parafoveal vasculature and structure in patients with LHON may become a useful biomarker with which to monitor disease progression, evaluate any therapeutic efficacy, and clarify the pathophysiology of the disease.

\section{Statement of Ethics}

The study was approved by the Ethics Committee of the Eye and ENT Hospital of Fudan University (no. ky2012-037) and was conducted ethically in accordance with the World Medical Association Declaration of Helsinki. Subjects gave their written informed consent, and the study protocol was approved by the institute's committee on human research.

\section{Conflict of Interest Statement}

The authors have no proprietary or commercial interests in any of the materials discussed in this article.

\section{Funding Sources}

The study was funded by the National Natural Science Foundation of China National Youth Project (81800846).

\section{Author Contributions}

Haohao Zhu and Min Wang had full access to all of the data and take responsibility for the integrity of the data and the accuracy of the data analyses. Haohao Zhu and Min Wang conceptualized and designed the study. All authors involved in the acquisition, analysis, or interpretation of data. Jian Yu and Huan Xu drafted the manuscript. Haohao Zhu and Min Wang critically revised the manuscript for important intellectual content. Jian $\mathrm{Yu}$ involved in statistical analysis. Haohao Zhu and Min Wang provided the administrative, technical, or material support. Haohao Zhu and Min Wang supervised the study.

\section{Data Availability Statement}

The datasets used and/or analyzed during the current study are available from the corresponding author on reasonable request.

\section{References}

1 Carelli V, Ross-Cisneros FN, Sadun AA. Mitochondrial dysfunction as a cause of optic neuropathies. Prog Retin Eye Res. 2004;23(1): 53-89.

2 Yu-Wai-Man P, Griffiths PG, Chinnery PF. Mitochondrial optic neuropathies disease mechanisms and therapeutic strategies. Prog Retin Eye Res. 2011;30(2):81-114.

3 Carelli V, La Morgia C, Iommarini L, Carroccia R, Mattiazzi M, Sangiorgi S, et al. Mitochondrial optic neuropathies: how two genomes may kill the same cell type? Biosci Rep. 2007;27:173-84.

4 Leber T. Ueber hereditäre und congenital-angelegte Sehnervenleiden. Graefe's Arhiv für Ophthalmologie. 1871;17(2):249-91.

5 Newman NJ. Hereditary optic neuropathies: from the mitochondria to the optic nerve. Am J Ophthalmol. 2005;140:511-7.

6 Balducci N, Savini G, Cascavilla ML, La Morgia C, Triolo G, Giglio R, et al. Macular nerve fibre and ganglion cell layer changes in acute Leber's hereditary optic neuropathy. Br J Ophthalmol. 2016;100(9):1232-7.

Changes in Retinal Perfusion in Leber's Hereditary Optic Neuropathy
7 Barboni P, Carbonelli M, Savini G, Ramos C, Carta A, Berezovsky A, et al. Natural history of Leber's hereditary optic neuropathy: longitudinal analysis of the retinal nerve fiber layer by optical coherence tomography. Ophthalmology. 2010;117(3):623-7.

8 Nikoskelainen EK, Huoponen K, Juvonen V, Lamminen T, Nummelin K, Savontaus ML. Ophthalmologic findings in Leber hereditary optic neuropathy, with special reference to mtDNA mutations. Ophthalmology. 1996; 103:504-14.

9 Nikoskelainen E, Hoyt WF, Nummelin K. Ophthalmoscopic findings in Leber's hereditary optic neuropathy. II. The fundus findings in the affected family members. Arch Ophthalmol. 1983;101:1059.

10 Nikoskelainen E, Hoyt WF, Nummelin K, Schatz H. Fundus findings in Leber's hereditary optic neuroretinopathy. III. Fluorescein angiographic studies. Arch Ophthalmol. 1984;102:981.

11 Jia Y, Tan O, Tokayer J, Potsaid B, Wang Y, Liu JJ, et al. Split-spectrum amplitude-decorrelation angiography with optical coherence tomography. Opt Express. 2012;20(4):4710.
12 Yu J, Gu R, Zong Y, Xu H, Wang X, Sun X, et al. Relationship between retinal perfusion and retinal thickness in healthy subjects: an optical coherence tomography angiography study. Invest Ophthalmol Vis Sci. 2016;57:OCT204.

13 Yu J, Jiang C, Wang X, Zhu L, Gu R, Xu H, et al. Macular perfusion in healthy chinese: an optical coherence tomography angiogram study. Invest Ophthalmol Vis Sci. 2015;56(5): 3212.

14 Huang Y, Zhou L, Zhangbao J, Cai T, Wang B, Li X, et al. Peripapillary and parafoveal vascular network assessment by optical coherence tomography angiography in aquaporin-4 antibody-positive neuromyelitis optica spectrum disorders. Br J Ophthalmol. 2019; 103(6):789-96.

15 Yu J, Xiao K, Huang J, Sun X, Jiang C. Reduced retinal vessel density in obstructive sleep apnea syndrome patients: an optical coherence tomography angiography study. Invest Ophthalmol Vis Sci. 2017;58:3506.

16 Jia Y, Morrison JC, Tokayer J, Tan O, Lombardi L, Baumann B, et al. Quantitative OCT angiography of optic nerve head blood flow. Biomed Opt Express. 2012;3:3127. 
17 Garway-Heath DF, Poinoosawmy D, Fitzke FW, Hitchings RA. Mapping the visual field to the optic disc in normal tension glaucoma eyes. Ophthalmology. 2000;107:1809-15.

18 Sharkawi E, Oleszczuk JD, Holder GE, Raina J. Clinical and electrophysiological recovery in Leber hereditary optic neuropathy with G3460A mutation. Doc Ophthalmol. 2012; 125:71-4.

19 Wang X, Jia Y, Spain R, Potsaid B, Liu JJ, Baumann B, et al. Optical coherence tomography angiography of optic nerve head and parafovea in multiple sclerosis. Br J Ophthalmol. 2014;98(10):1368-73.

20 Balducci N, Ciardella A, Gattegna R, Zhou Q, Cascavilla ML, La Morgia C, et al. Optical coherence tomography angiography of the peripapillary retina and optic nerve head in dominant optic atrophy. Mitochondrion. 2017;36: 60-5.

21 Balducci N, Cascavilla ML, Ciardella A, La Morgia C, Triolo G, Parisi V, et al. Peripapillary vessel density changes in Leber's hereditary optic neuropathy: a new biomarker. Clin Experiment Ophthalmol. 2018;46(9):105562.
22 Borrelli E, Balasubramanian S, Triolo G, Barboni P, Sadda SR, Sadun AA. Topographic macular microvascular changes and correlation with visual loss in chronic leber hereditary optic neuropathy. Am J Ophthalmol. 2018;192:217-28.

23 Kousal B, Kolarova H, Meliska M, Bydzovsky J, Diblik P, Kulhanek J, et al. Peripapillary microcirculation in Leber hereditary optic neuropathy. Acta Ophthalmol. 2019;97:e71-6.

24 Matsuzaki M, Hirami Y, Uyama H, Kurimoto Y. Optical coherence tomography angiography changes in radial peripapillary capillaries in Leber hereditary optic neuropathy. Am J Ophthalmol Case Rep. 2018;9:51-5.

25 Carelli V, La Morgia C, Valentino ML, Barboni P, Ross-Cisneros FN, Sadun AA. Retinal ganglion cell neurodegeneration in mitochondrial inherited disorders. Biochim Biophys Acta. 2009;1787:518-28.

26 Asanad S, Tian JJ, Frousiakis S, Jiang JP, Kogachi K, Felix CM, et al. Optical Coherence tomography of the retinal ganglion cell complex in Leber's hereditary optic neuropathy and dominant optic atrophy. Curr Eye Res. 2019;44:638-44.

27 Li B, Freeman RD. Neurometabolic coupling between neural activity, glucose, and lactate in activated visual cortex. J Neurochem. 2015; 135(4):742-54
28 Pan BX, Ross-Cisneros FN, Carelli V, Rue KS, Salomao SR, Moraes-Filho MN, et al. Mathematically modeling the involvement of axons in Leber's hereditary optic neuropathy. Invest Ophthalmol Vis Sci. 2012;53:7608.

29 Guy J, Feuer WJ, Porciatti V, Schiffman J, Abukhalil F, Vandenbroucke R, et al. Retinal ganglion cell dysfunction in asymptomatic G11778A: Leber hereditary optic neuropathy. Invest Ophthalmol Vis Sci. 2014;55(2):841.

30 Zabel P, Kaluzny JJ, Wilkosc-Debczynska M, Gebska-Toloczko M, Suwala K, Zabel K, et al. Comparison of retinal microvasculature in patients with Alzheimer's disease and primary open-angle glaucoma by optical coherence tomography angiography. Invest Ophthalmol Vis Sci. 2019;60:3447-55.

31 Kwapong WR, Ye H, Peng C, Zhuang X, Wang J, Shen M, et al. Retinal Microvascular impairment in the early stages of Parkinson's disease. Invest Ophthalmol Vis Sci. 2018;59: 4115-22.

32 Lu P, Xiao H, Liang C, Xu Y, Ye D, Huang J. Quantitative analysis of microvasculature in macular and peripapillary regions in early primary open-angle glaucoma. Curr Eye Res. 2020;45:629-35. 